Josée-Anne Gouin

Université Laval

Christine Hamel

Université Laval

Érick Falardeau

Université Laval

\section{L'analyse des savoirs mobilisés par des étudiantes-stagiaires en enseignement dans leur pratique réflexive}

Circulation of knowledge of student-trainees in initial training in secondary education

doi: $10.18162 / f p .2018 .454$
L'articulation théorie-pratique mise de l'avant dans les programmes de formation universitaires à l'enseignement apporte son lot de défis (Bullough et Draper, 2004), dont celui du développement de la pratique réflexive (Comité d'agrément des programmes de formation à l'enseignement

[CAPFE], 2015). La présente recherche s'est intéressée à cette articulation théorie-pratique à l'intérieur de trois cours universitaires associés à la sixième session du baccalauréat en enseignement secondaire : le troisième stage de cinq semaines, un cours de didactique du français et un cours

de gestion de classe. L'analyse des travaux de quatre étudiantes a permis d'identifier les sources de savoirs qu'elles ont mobilisées ainsi que le processus réflexif qu'elles ont emprunté.

\section{Mots-clés}

CArticulation théorie-pratique, formation initiale, pratique réflexive.

\section{Abstract}

The practical theory articulation prescribed by the university training programs in education brings its challenges (Bullough \& Draper, 2004), including the development of reflective practice (CAPFE, 2015). The present research focused on this practical theory articulation within three university courses associated with the sixth session of the baccalauréat in secondary education: the third five-week course, a course in French didactics and a course in management of class. We analyzed the work of four female students. The results identify the sources of knowledge nominated by the students and the reflective process they have taken.

\section{Keywords}

Articulation practical theory, teacher education, reflexive practice.

\section{Contexte et problématique}

L'une des principales visées du référentiel La formation à l'enseignement - Les orientations - Les compétences professionnelles (Ministère de l'Éducation du Québec [MEQ], 2001) concerne la professionnalisation des futurs enseignants. Pour assurer ce développement, chacune des universités québécoises propose des programmes de formation favorisant l'alternance entre les cours et les stages.

À l'Université Laval, lors de leur troisième année d'études, les étudiants-stagiaires en enseignement au secondaire effectuent un stage de prise en charge (50\% de la tâche d'enseignement) d'une durée de cinq semaines, supervisé par un enseignant associé et un superviseur universitaire. Ce stage se déroule en alternance avec quatre autres cours : la didactique du français, les aspects sociaux de l'éducation, la gestion de la classe et l'évaluation des apprentissages. De nombreux défis entourent l'articulation théorie-pratique à l'intérieur des programmes de formation en enseignement. Nous avons analysé trois travaux écrits de quatre étudiantes-stagiaires afin de connaitre les types de savoirs qui émergent des cours universitaires et du stage. Cela a permis d'identifier la source des savoirs mobilisés dans leur discours écrit. Nous voulions également analyser la place que prend la pratique réflexive des étudiantsstagiaires dans ces travaux. Les travaux écrits proviennent de la didactique du français, de la gestion de classe et du stage III. 


\section{L'articulation théorie-pratique en formation initiale : des défis à surmonter dans l'accompagnement des étudiants- stagiaires}

Le processus de professionnalisation des étudiants-stagiaires qui passe par l'articulation théoriepratique est soutenu, au Québec, par une formation en alternance (MEQ, 2001) : quelque 700 heures de stage sont réparties sur les quatre années de formation du baccalauréat.

L'articulation théorie-pratique se réalise à travers la pratique réflexive (Korthagen, 2010). Selon Korthagen (2010), l'articulation théorie-pratique qui est mise de l'avant dans les programmes de formation universitaires se réalise difficilement parce que les étudiants-stagiaires restent dans une analyse intuitive de leur pratique, qui n'est pas assez alimentée par des savoirs véhiculés à l'université lorsque les étudiants sont en stage ou qui n'est pas assez alimentée par des savoirs pratiques lorsque les étudiants ont des cours académiques. Ainsi, les étudiants-stagiaires éprouvent des difficultés à prendre du recul par rapport à la pratique qu'ils observent chez leur enseignant associé (Bullough et Draper, 2004). Par ailleurs, les étudiants-stagiaires qui se sentent « efficaces » dans le pilotage d'une situation d'enseignement et d'apprentissage ne remettront pas en question les pratiques pédagogiques adoptées, surtout si elles correspondent à celles de leur enseignant associé (Serres, Ria et Adé, 2004). Enfin, les savoirs enseignés à l'université peuvent être évacués par les étudiants-stagiaires lorsqu'ils arrivent en stage, surtout si l'enseignant associé et le superviseur universitaire y réfèrent peu de manière explicite (Korthagen et Vasalos, 2005).

À cet égard, l'accompagnement des étudiants-stagiaires dans le modèle de la triade (enseignant associé, superviseur universitaire et étudiant-stagiaire), malgré certains avantages documentés, comporte son lot de défis. Des recherches (Bullough et Draper, 2004; Harrison, Dymoke et Pell, 2006) ont montré que les formateurs de stagiaires, les enseignants associés et les superviseurs universitaires ont une perception de leurs fonctions professionnelles qui ne correspond pas aux attentes prescrites par le modèle de la triade. Du côté des superviseurs, c'est le rôle de médiateur qui semble poser problème : une médiation difficile entre les attentes universitaires et celles du milieu scolaire ou entre l'enseignant et l'étudiant-stagiaire lorsque des conflits surviennent (Desbiens, Spallanzani et Borges, 2013). Du côté des enseignants associés, ce sont certaines pratiques d'accompagnement des étudiants-stagiaires qui demeurent nébuleuses, notamment lorsque des enseignants encouragent les étudiants-stagiaires à seulement reproduire leurs pratiques professionnelles (Clarke et Collins, 2007). En fait, c'est principalement lorsqu'il est question de l'articulation théorie-pratique chez l'étudiant-stagiaire que les pratiques d'accompagnement des deux formateurs demeurent floues (Portelance, Gervais, Lessard et Beaulieu, 2008). Ayant très peu de contacts avec les professeurs et chargés de cours universitaires (Tardif et Jobin, 2014), les superviseurs universitaires ne connaissent pas nécessairement le contenu des cours offerts à l'université, et la place qu'y occupent les plus récentes recherches et les savoirs théoriques. De plus, dans certains cas, il y a une crainte importante de faire de l'ingérence dans le travail des enseignants associés (Desbiens, Spallanzani et Borges, 2013) : comment favoriser les liens théoriepratique sans porter un jugement sur le travail des enseignants associés auprès de leurs élèves? 


\section{La pratique réflexive pour favoriser l'articulation théorie-pratique}

Dès 1994, le ministère de l'Éducation du Québec (MEQ) souligne l'importance de la pratique réflexive dans la formation initiale. En 2001, le MEQ insère la pratique réflexive comme une compétence professionnelle dans son référentiel de formation : "réfléchir sur sa pratique (analyse réflexive) et réinvestir les résultats de sa réflexion dans l'action » (p. 127). Néanmoins, la théorisation de la pratique réflexive comme une activité individuelle intrapersonnelle (Sharples, 2005) limite son déploiement : le non-accompagnement de l'étudiant-stagiaire dans sa pratique réflexive peut nuire à la pertinence des questions qu'il se pose et à la cohérence des réponses qu'il y apporte (Korthagen et Vasalos, 2005).

L'articulation théorie-pratique est nécessaire pour la création de savoirs professionnels (dans un processus de professionnalisation), dans la mesure où la pratique réflexive des étudiants-stagiaires est soutenue et enrichie par eux-mêmes, leurs pairs et leurs formateurs (Avalos, 2011; Clarke, 2006). Cependant dans la littérature, nous retrouvons une pluralité de définitions qui opérationnalisent le concept de pratique réflexive : certains auteurs parlent de processus cognitifs (ou de niveaux) qui permettent une réflexion en cours et sur l'action (Calderhead, 1989; Donnay et Charlier, 2006; Jay et Johnson, 2002; Schön, 1983). D’autres présentent la pratique réflexive comme une succession d'étapes dans un processus d'apprentissage pour décortiquer, analyser une action et proposer des solutions à réinvestir (Dewey, 1938; Rogers, 2002). D'autres encore y accolent la définition d'un cycle d'apprentissage sous forme «d'étapes spiralaires » : c'est-à-dire que les étapes pourront se réaliser dans un ordre non défini et se termineront par le réinvestissement dans une nouvelle situation (Korthagen, 1985). Il revient donc aux programmes universitaires de définir la pratique réflexive et de s'approprier un cadre pour éviter qu'elle reste implicite dans la formation des étudiants-stagiaires. De plus, l'importance de l'interaction entre le formateur et l'étudiant-stagiaire est primordiale dans le développement de la pratique réflexive puisque la plupart des études ont montré qu'il ne s'agit pas de vivre une expérience en stage pour que l'étudiant-stagiaire mobilise cette compétence. La pratique réflexive doit donc être accompagnée en formation initiale (Butke, 2006; Korthagen, 2016; Mann, Gordon et MacLeod, 2009; Rogers, 2002). Les formateurs, par leurs questionnements, ont la responsabilité de soutenir le développement professionnel des étudiants-stagiaires afin que la compréhension de l'expérience vécue puisse transformer la pratique (Butke, 2006; Mann et al., 2009).

\section{Cadre conceptuel et d'analyse}

\section{L'articulation théorie-pratique soutenue par la pratique réflexive}

Bien que plusieurs interprétations de l'alternance théorie-pratique semblent vouloir coexister en formation à l'enseignement, notre étude ne s'inscrit pas dans un cadre applicationniste qui demande de reproduire ce qui est appris à l'université. Elle s'attarde plutôt sur une interfécondation tenant compte des contraintes des deux milieux pour en arriver à former un futur enseignant capable de théoriser sa pratique à partir de ses expériences d'enseignement. Dans ce modèle, l'apport des formateurs universitaires est crucial puisqu'ils doivent soutenir les étudiants-stagiaires afin que ceux-ci saisissent davantage les opportunités d'apprentissage et de développement professionnel en percevant mieux ce qui se passe dans la classe. Ils soutiennent les capacités des stagiaires à faire les liens entre ce qu'ils ont 
compris (niveau schéma) pour les faire passer au niveau théorique (théorisation de l'activité). La mise en œuvre de l'articulation théorie-pratique repose alors en grande partie sur la capacité des étudiantsstagiaires à adopter une pratique réflexive (Korthagen, 2010).

Le cadre retenu pour comprendre l'alternance théorique à travers la pratique réflexive des étudiantsstagiaires s'appuie sur le modèle ALACT (Korthagen et Vasalos, 2005) qui présente des étapes. Ce modèle repose sur la prémisse que les enseignants réfléchissent naturellement à leurs expériences professionnelles. Korthagen et Vasalos (2005) mentionnent que la pression du travail encourage souvent les enseignants à mettre l'accent sur l'obtention d'une solution rapide à leurs problèmes en classe - solution rapide pour un problème pratique - plutôt que de mettre en lumière les causes des problèmes vécus par leurs élèves. L'enseignant n'a plus l'habitude d'examiner ses stratégies, et encore moins de les analyser (Korthagen et Vasalos, 2005). Comme enseignant associé, il devient parfois difficile pour lui d'accompagner son étudiant-stagiaire en ce sens tellement certaines pratiques sont ancrées et difficiles à expliquer. Ainsi, la réflexion structurée s'avère importante pour soutenir le développement de la pratique réflexive dans un contexte d'alternance. L'étudiant-stagiaire entre alors dans un processus réflexif qui va plus loin que la résolution spontanée de problèmes d'enseignement. Habituellement, il s'initie à ce processus d'analyse approfondie de son agir professionnel à travers un travail d'écriture ou de discussion médiatisé par un formateur. Le modèle élaboré par Korthagen et Vasalos (2005) structure, sans la rendre linéaire, la réflexion sur l'action en mettant en lumière cinq étapes : 1) l'action qui représente une pratique quotidienne en classe ou en rencontre avec ses collègues par exemple; 2) le retour sur l'action qui peut prendre la forme d'une description de l'action vécue préalablement; 3) l'identification des aspects essentiels de la situation qui a été décrite; 4) la réflexion et la création de méthodes alternatives pour résoudre le ou les problèmes en analysant les conséquences pour chaque méthode; 5) le réinvestissement dans l'action (mise à l'essai) du jugement professionnel des différentes méthodes réfléchies à la précédente étape.

\section{Réflexion/création de méthodes alternatives}

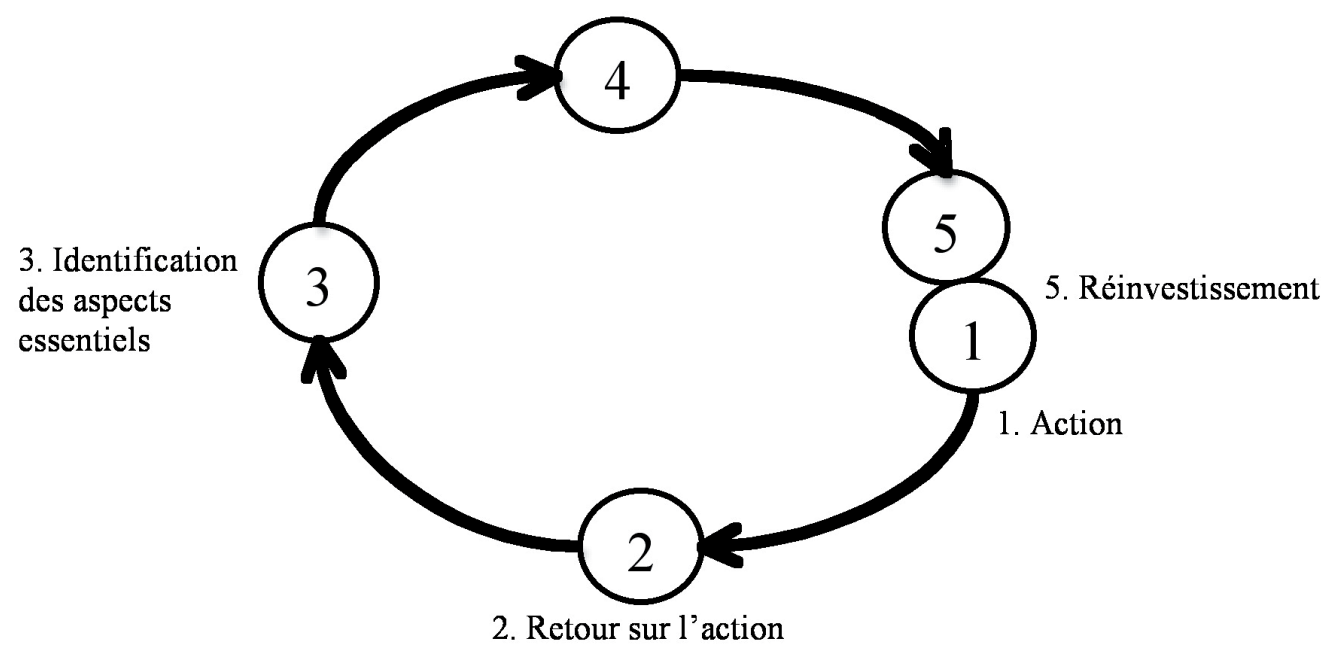

Figure 1

Modèle ALACT de réflexion sur la pratique (adapté de Korthagen et Vasalos, 2005)

(Action - Looking back on the action - Awareness of essential aspects - Creating alternative methods of action - Trial). 
Le modèle de Korthagen et Vasalos suggère aux formateurs un rôle de médiateurs dans l'exercice de la pratique réflexive. Par exemple, au moment de la phase 2 - Retour sur l'action, les formateurs peuvent poser différentes questions pour amener l'étudiant-stagiaire à mettre sa pratique à distance à l'aide de savoirs théoriques ou pratiques : quel était le contexte de la tâche demandée? Était-il clairement posé? Comment les élèves ont-ils réalisé leur travail? Comment les y as-tu guidés? Quels problèmes de compréhension ont rencontrés les élèves? Quelles inférences leur ont posé problème? Comment les as-tu questionnés pour prendre la mesure de leurs difficultés? Comment as-tu ajusté ton intervention devant ces difficultés?

\section{Les sources de savoirs}

Pour soutenir l'articulation théorie-pratique et la pratique réflexive en formation initiale à l'enseignement, les formateurs doivent mieux comprendre quelles peuvent être les sources de savoir auxquelles les futurs enseignants ont accès. Nous utilisons le cadre proposé par Buysse (2011) pour caractériser ces sources de savoirs, tout en étant conscients qu'une grande variété de typologies de savoirs est disponible dans la littérature scientifique. Les savoirs professionnels se construisent lorsque les contenus théoriques (savoirs à enseigner et savoirs pour enseigner) sont mis en relation avec la pratique. La création de savoirs professionnels est alors rendue possible grâce à l'intégration, par l'étudiant-stagiaire, des savoirs de référence aux savoirs d'expérience. C'est ce que Buysse (2011) nomme l'articulation théorie-pratique soutenue par la pratique réflexive. Il distingue cinq sources : 1) les savoirs scientifiques sont proposés aux étudiants comme des références à des données scientifiques. Ces savoirs se fondent sur une littérature spécialisée produite par des chercheurs. 2) Les savoirs académiques, eux, sont enseignés par l'institution universitaire. Ils proviennent de la recherche et ont été didactisés. 3) Les savoirs prescriptifs sont des orientations pour agir en fonction des attentes de la société et de l'institution : par exemple, les cadres d'évaluation ministériels, les épreuves ministérielles ou le Programme de formation de l'école québécoise. 4) Les savoirs de la pratique sont issus des échanges avec les enseignants associés et les superviseurs universitaires et proposés comme pertinents ou efficaces. Enfin, 5) les savoirs de vulgarisation donnent des pistes concrètes, des outils, des méthodes et ils font partie d'ouvrages non scientifiques tels que des sites internet d'enseignants, des manuels de classe et des articles de presse.

\section{Question de recherche}

Dans le contexte où nous sommes préoccupés par une meilleure articulation théorie-pratique, nous avons cherché à savoir si les étudiants-stagiaires mobilisent les savoirs pratiques et scientifiques dans leurs cours et dans leurs stages. Lors de la troisième année de formation (sixième session), nous nous sommes intéressés aux sources de savoirs de référence véhiculées à l'intérieur de différents travaux pour quatre stagiaires inscrites en enseignement du français au secondaire, ainsi qu'à l'articulation des étapes du modèle ALACT. Nos questions de recherche sont les suivantes :

1) Quelles sont les sources de savoirs mobilisées dans le discours écrit des quatre étudiantes?

2) Comment s'articulent les différentes étapes de la pratique réflexive des participantes selon le modèle ALACT en relation avec les savoirs mobilisés? 


\section{Méthodologie}

Les étudiants du groupe d'un cours de didactique du français au baccalauréat en enseignement du français au secondaire ont été invités par courriel à participer à cette recherche. Quatre étudiantes parmi les 32 inscrits ont signifié leur intérêt. Elles sont identifiées par les lettres A, B, C, et D afin de préserver leur anonymat. Une analyse descriptive, décrite plus loin, a été réalisée à partir de trois travaux écrits demandés dans trois cours de la sixième session. Ces trois travaux ont été ciblés puisque les auteurs sont professeurs responsables de ces trois cours et que des efforts importants ont été investis pour soutenir l'articulation théorie-pratique au sein de ces cours depuis quelques années.

En didactique du français, une partie d'un travail d'intégration portant sur une situation d'enseignementapprentissage (SEA) vécue en stage a été analysée qualitativement. Les étudiants-stagiaires analysaient la planification et le pilotage de cette situation. Différentes pistes de questionnements leur étaient proposées et ils choisissaient celles qui correspondaient le mieux à leur SEA. Par exemple, identifier et expliquer un décalage entre votre planification initiale et votre prise en charge. Quelles différences avez-vous observées sur le plan des contenus, des apprentissages et de vos interventions? Expliquez-en les causes. Une consigne plus générale leur demandait également de revenir de manière réflexive sur leurs interventions dans la perspective de la didactique de leur discipline.

En gestion de classe, une analyse des contributions partagées sur un forum de coélaboration de connaissances (Scardamalia, 2004) a été réalisée. Coélaborer des connaissances signifie qu’il faut aller au-delà du partage du savoir : interagir ensemble (informer, suggérer, réfuter, etc.) dans le but d'apprendre. Les étudiants-stagiaires pouvaient contribuer sur tous les sujets qu'ils jugeaient pertinents en respectant le thème de la gestion de classe au sens théorique du terme, soit la gestion et la supervision des interactions afin de créer un climat propice pour l'apprentissage.

Dans le stage III, des journaux professionnels ont été récupérés. Ces journaux respectaient une structure clairement décrite dans les documents de stage : 1) décrire une situation réelle concrète, satisfaisante ou problématique, qui a retenu l'attention et identifier un problème ${ }^{1}$ sous forme d'interrogation ou de préoccupation; 2) analyser la situation en mettant en relation les éléments en cause et en expliquant les éléments du contexte (déconstruction); 3) établir des liens avec des savoirs théoriques susceptibles de favoriser la compréhension de la situation énoncée. Ces savoirs théoriques doivent être issus des contenus de cours suivis à l'université, des assises théoriques du stage, d'ouvrages spécialisés en éducation ainsi que des savoirs et savoir-faire formalisés ${ }^{2}$ par l'enseignant associé; 4) réaliser une synthèse permettant de tirer des conclusions, de dégager une piste de solution ou des éléments de savoirs pratiques témoignant de la construction d'un savoir professionnel (reconstruction).

Pour cibler les savoirs de référence à l'intérieur des trois travaux, une analyse de contenu par distinction de catégories (Krippendorff, 2004) a été menée. Afin de coder les travaux des étudiantes, une grille de codage a été élaborée à l'intérieur de laquelle les cinq sources de savoir ont été représentées. Il s'agit donc d'un plan de codage préétabli en fonction du cadre conceptuel. Les unités de sens pouvaient être une phrase, quelques phrases et même dans certains cas, des paragraphes, en autant quelles respectent la catégorie des savoirs mobilisés : scientifiques, académiques, de vulgarisation, prescriptifs et de la pratique. Par exemple, l'étudiante B a cité un extrait d'une revue en littérature : « En fait, il aurait fallu que jenseigne aux élèves les raisons pour lesquelles ils apprennent une stratégie et pourquoi elle leur sera utile, 
sans quoi ces derniers risquent de démontrer peu de motivation et faire peu d'efforts dans l'apprentissage des stratégies (référence à la revue) ». Cet énoncé a été classé dans la catégorie « savoir académique » puisque le texte qu'elle citait ne présentait pas de données de recherche, mais didactisait des savoirs.

Afin d'analyser les étapes empruntées par les étudiants dans leur pratique réflexive, une seconde analyse de contenu par distinction de catégories a été réalisée. Après une lecture des travaux, les unités de sens (phrases, paragraphes) ont été relevées. Ces unités représentaient l'une des étapes de la pratique réflexive selon le modèle ALACT. Ensuite, dans chaque travail, ces unités de sens ont été numérotées afin d'en comprendre le déroulement chronologique. Deux validations interjuges ont été menées comme le recommandent Miles et Huberman (2003) avec un taux de 98 \% d'accord entre les deux codeurs.

\section{Les résultats}

Nous présenterons les savoirs de référence mobilisés par les quatre étudiants-stagiaires à l'intérieur des trois travaux : le travail d'intégration en didactique du français, le journal professionnel ainsi que le forum de coélaboration de connaissances. Par la suite, nous aborderons l'analyse de l'articulation du modèle ALACT. Dans cette deuxième section d'analyse, nous présenterons un résumé sommaire des résultats obtenus : pour cette raison, seuls les tableaux les plus parlants seront explicités.

\section{Les savoirs de référence mobilisés par les étudiants}

\section{Dans le travail d'intégration (nombre de savoirs=29) (didactique du français)}

La figure suivante présente les savoirs de référence mobilisés par les quatre étudiantes dans leur travail d'intégration en didactique du français :

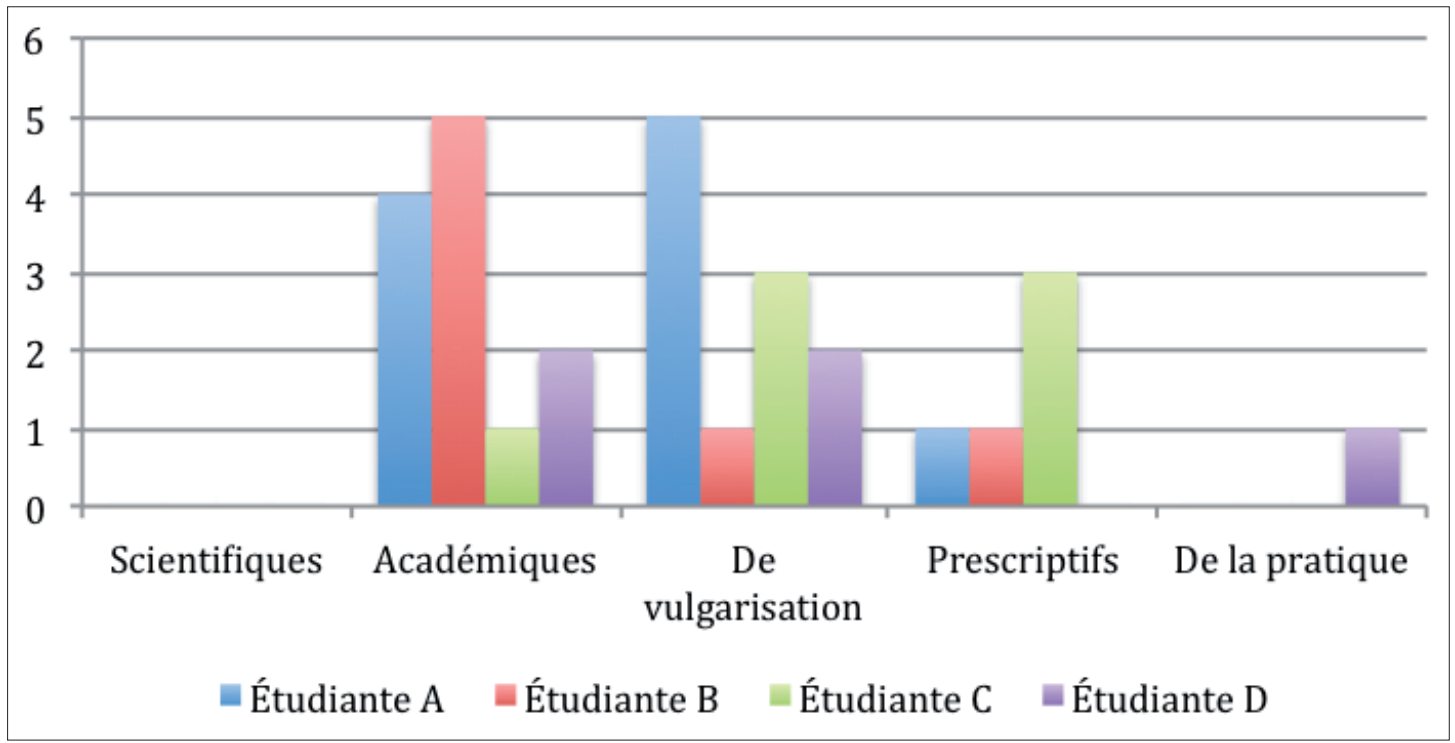

Figure 2

Les sources de savoirs de référence pour le travail d'intégration en didactique du français. 
À l'intérieur de cette figure, les savoirs de source académique et de vulgarisation sont ceux qui dominent (80\%). Par exemple, les étudiantes abordent la motivation scolaire en s'appuyant sur un texte de nature académique distribué dans un cours universitaire : «[...] pour que les élèves soient motivés à s'investir [...], il est essentiel que la situation d'apprentissage présente un réel défi intellectuel [...]. Cela correspond aux dix conditions de Viau (2000)" (étudiante A).

Régulièrement, les étudiantes vulgarisent en référant à des contenus de cours universitaires, mais n'appuient pas leurs propos à l'aide de travaux de recherche. Par exemple, l'étudiante A fait référence au contenu de son cours de gestion de classe à l'intérieur de son travail en didactique, mais sa source est absente : «Or, cela est bien connu, moins l'enseignant doit gérer des comportements dérangeants, plus il peut consacrer du temps de qualité à l'enseignement de sa matière et plus les élèves sont susceptibles de faire des apprentissages significatifs». Les savoirs scientifiques sont absents de son propos et ceux provenant de la pratique sont peu présents. L'étudiante $\mathrm{D}$, de son côté, s'est référée à un conseil de son enseignant (savoir de la pratique) pour appuyer son choix de stratégie d'enseignement : «[...] j’ai décidé de faire une brève présentation théorique de façon magistrale en lien avec le résumé, entre autres parce que mon enseignant associé me le conseillait fortement ».

La nature des savoirs nommés a été analysée. Létudiante $\mathrm{A}$ a fait ressortir davantage de savoirs que les trois autres étudiantes, et ils sont de nature plus diversifiée. Les savoirs qu'elle nomme réfèrent aux stratégies d'enseignement qu'elle a exploitées en classe, aux stratégies d'apprentissage des élèves, à leur motivation face aux différentes tâches demandées ainsi qu'à la mobilisation de ses compétences professionnelles. De plus, elle a fait un diagnostic de ses élèves, relevant leurs forces et leurs faiblesses, avant le début de la situation d'enseignement-apprentissage (SEA) (faisant l'objet de l'analyse du travail en didactique), afin de faire les "meilleurs choix possibles " dans les stratégies d'enseignement et d'apprentissage qu'elle met en place. Cela correspond à la phase 3 du modèle ALACT : identifications des aspects. L'étudiante B n'a pas fait de diagnostic par rapport aux forces et aux difficultés des élèves avant, pendant ou après la SEA. Les sources qu'elle cite sont parfois incomplètes. Létudiante $\mathrm{C}$ réfère surtout aux savoirs de source prescriptive : le référentiel des compétences professionnelles (MEQ 2001). Elle vulgarise les savoirs étudiés dans ses cours universitaires et elle fait un diagnostic des élèves avant la SEA afin d'appuyer ses choix pédagogiques. Finalement, l'étudiante D se sert de la situation d'enseignement-apprentissage pour faire un diagnostic des élèves à la toute fin : phase 3 du modèle ALACT, identification des aspects. À la suite de l'identification des forces et des faiblesses des élèves, elle n'appuie pas ses décisions professionnelles sur des savoirs : elle fait ses choix en fonction de ses croyances personnelles. Son texte présente d'ailleurs plusieurs « Je crois que... Je considère que...».

\section{Dans le journal professionnel (Stage III) (nombre de savoirs : 11)}

La figure 3 présente les sources de savoirs relevées dans le travail du journal professionnel réalisé au cours du stage III. Il est à noter que l'étudiante B a travaillé dans un forum de coélaboration de connaissances plutôt que de réaliser un journal professionnel. 


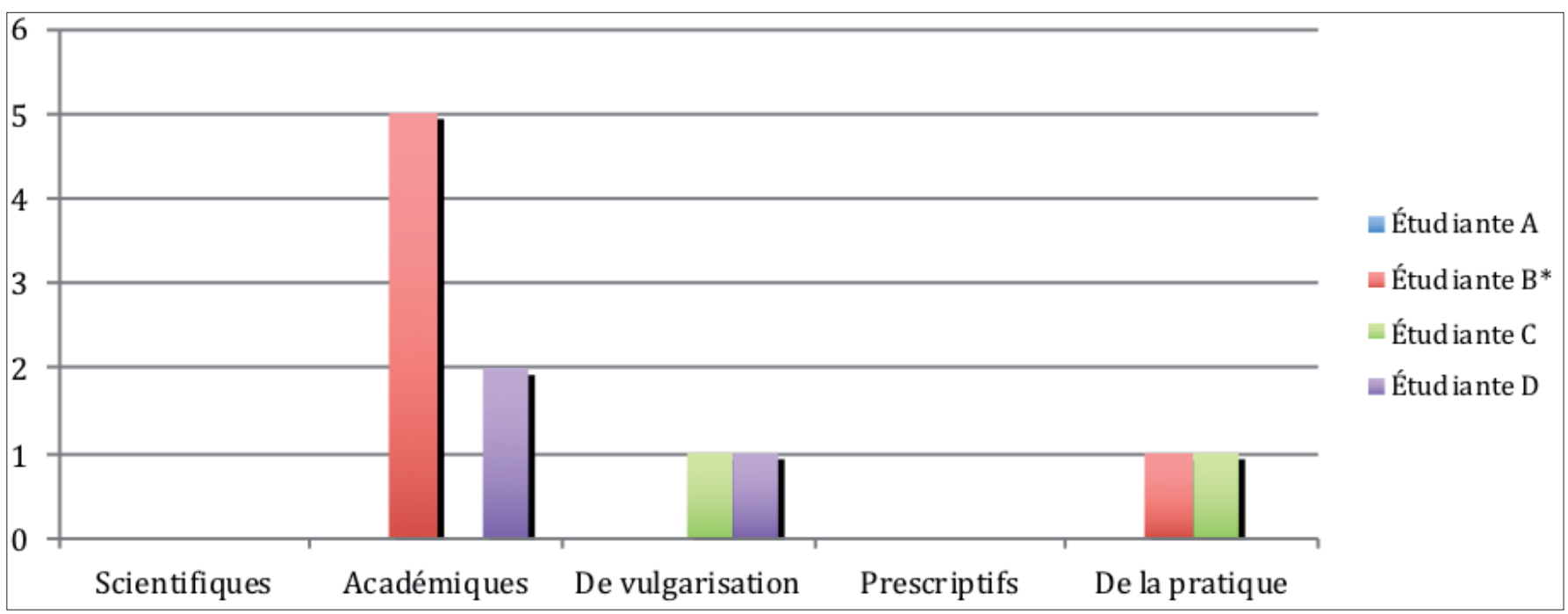

\section{Figure 3}

Les sources de savoirs de référence pour le journal professionnel en stage III.

Comme dans le travail d'intégration réalisé dans le cours de didactique du français, les savoirs scientifiques sont absents du journal professionnel. De plus, en comparant ce graphique avec celui du travail d'intégration, beaucoup moins de savoirs émergent du journal professionnel (11 occurrences par rapport à 29 pour le travail d'intégration). Le journal ne fait l'objet d'aucune rétroaction, aucun dialogue entre le formateur et le formé; il est simplement corrigé de façon sommative. Par exemple, dans le travail de didactique, l'étudiante A mobilisé des savoirs de plusieurs sources alors que ce n'est pas le cas dans son journal professionnel. L'étudiante B, celle qui a exploité un forum de discussion (Knowledge Forum), a mobilisé beaucoup plus de savoirs que les autres étudiantes. Les savoirs qu'elle nomme sont de nature variée et sont enrichis par les contributions de ses pairs. Le caractère dialogique du forum semble donc avoir un effet positif sur le nombre de savoirs convoqués dans l'analyse. À cela, ajoutons que cette étudiante aborde plusieurs thèmes qui la préoccupent (pour une longueur de travail similaire au journal professionnel), ce qui lui permet d'appuyer ses propos par des savoirs variés qui ont été exigés par sa superviseure de stage. Le contraste est important entre l'étudiante B et les trois autres, qui ont beaucoup moins recouru à des savoirs pour alimenter leur réflexion. 
Les sources de savoirs dans le forum de coélaboration de connaissances (Gestion de la classe) (nombre de savoirs: 15)

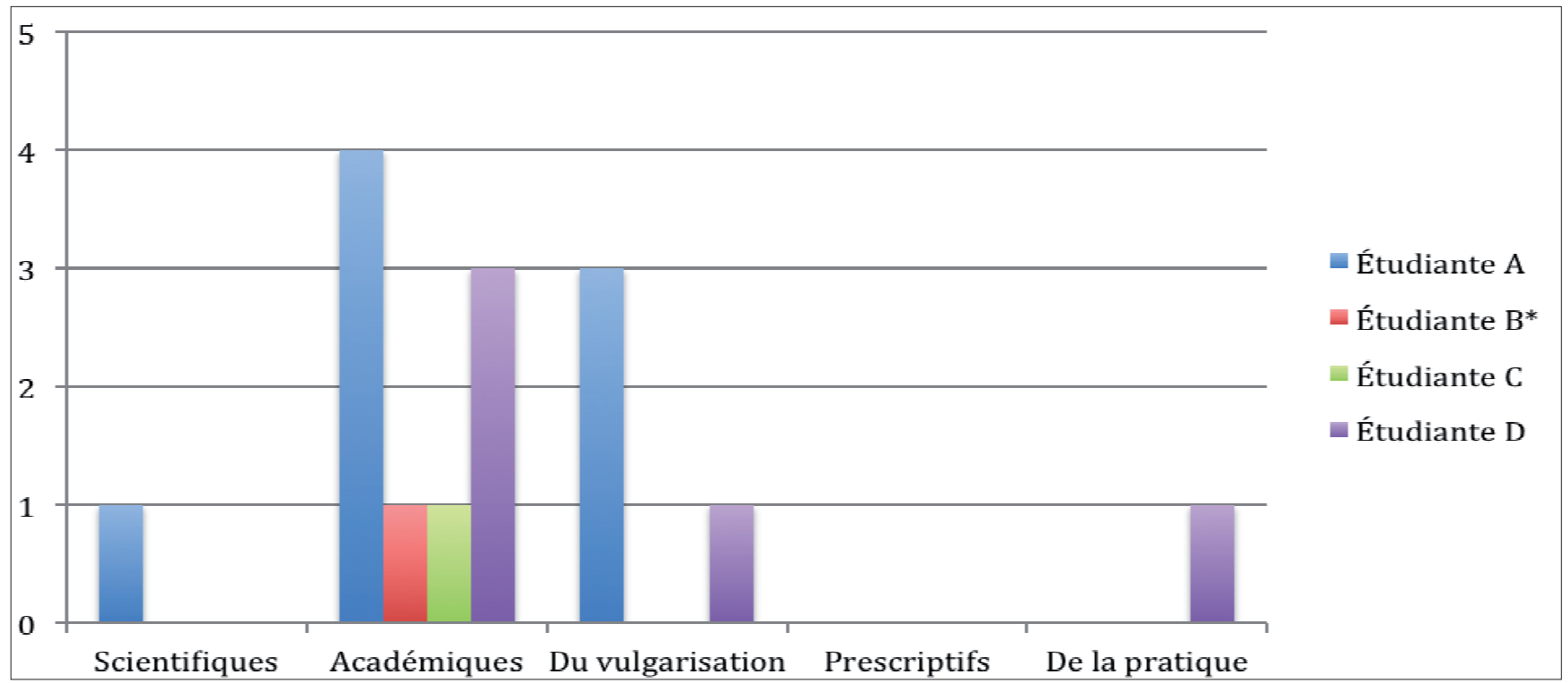

Figure 4

Les sources de savoirs de référence pour le forum de coélaboration de connaissances en gestion de classe.

Ici encore, les savoirs les plus fréquents sont de source académique. Par exemple : « [...] La différenciation pédagogique est une philosophie qui guide l'ensemble des pratiques pédagogiques et une façon d'exploiter les différences et d'en tirer avantage (MELS, 2006) » (étudiante B). Le seul savoir nommé provenant d'une source scientifique n'est pas explicité, seulement nommé après une réflexion incomplète : «[...] même si les résultats de la métaanalyse de John Hattie stipulent que la différenciation pédagogique n’influence pas les résultats des élèves, je ne peux pas en dire autant " (étudiante A). L'étudiante ne poursuit pas sa réflexion; il est alors difficile de comprendre ce qu'elle voulait exprimer à l'aide de cette source. Du côté de la pratique, un seul savoir est nommé : «[...] l'un des commentaires que l'on [en référence à son formateur] m'a donnés est de toujours inclure plus d'activités dans le plan de cours » (étudiante D). Pourtant les contributions des étudiantes portent sur la gestion de classe, un domaine qui ouvre la porte à de nombreuses expériences de la pratique (stages antécédents, expériences d'élèves, expériences nommées par les enseignants associés). Malgré son caractère dialogique, qui met les stagiaires en discussion autour des cas rapportés par chacun, le forum entraine peu les stagiaires à étayer leur réflexion à l'aide de savoirs, probablement parce que le dialogue est entretenu avec des pairs plutôt qu'avec un formateur qui pourrait proposer des pistes de réflexion appuyées par des savoirs. Comme nous l'avons vu plus haut, létudiante B a mobilisé davantage de savoirs lorsque ses formateurs l'ont questionnée dans le forum (en remplacement du journal professionnel). En somme, l'analyse des savoirs mobilisés dans les trois travaux mis en place pour favoriser l'articulation théorique pratique entre le stage et les cours suivis à l'université met en exergue la faible présence des savoirs scientifiques dans le discours des étudiantes. Les travaux qui exigent un dialogue avec les formateurs et les pairs mobilisent davantage de savoirs que ceux qui n'exigent aucune rétroaction durant leur élaboration. 


\section{L'articulation des étapes du modèle ALACT}

Nous avons repris le modèle de Korthagen et Vasalos (2005) pour illustrer l'articulation des étapes réalisées par chaque étudiante. Nous vous présentons un résumé des résultats.

\section{Étudiante A (pour les trois travaux)}

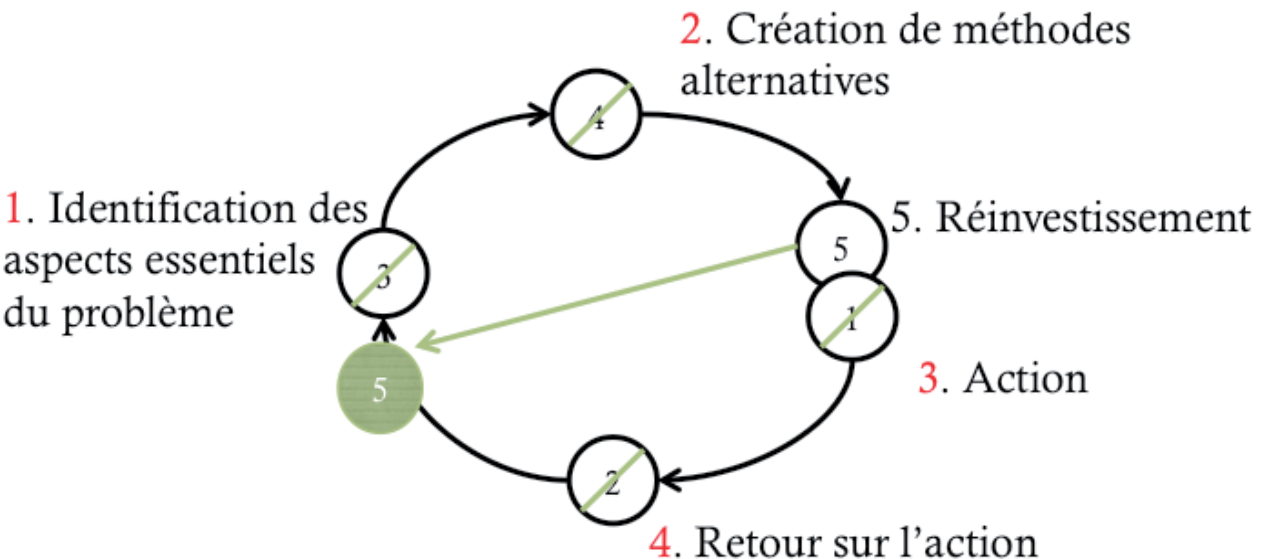

Figure 5

L'articulation des étapes du modèle ALACT pour l'étudiante A.

L'étudiante A, pour chacun de ses travaux, a vécu les étapes du modèle ALACT dans le même ordre pour ses trois travaux : comme première étape, elle a d'abord identifié d'éventuels problèmes en faisant un diagnostic de ses élèves avant l'élaboration de ses activités d'apprentissage (donc avant de passer à l'action) : "J'ai eu la chance de travailler avec des élèves extrêmement performants [...]. Par conséquent, pour que ces élèves soient motivés à s'investir, il était essentiel que les activités proposent des défis (Viau, 2000)». Comme deuxième étape, après chaque diagnostic des élèves, elle a proposé des solutions (des stratégies d'enseignement) qu'elle a appuyées sur des sources de savoirs académiques, de vulgarisation ou de prescription : "C'est alors que me vint l'idée d'enseigner différentes règles [...] par le biais d'une démarche active de découverte ». Elle cite alors une chercheure dans le domaine de l'enseignement de la grammaire pour justifier sa stratégie d'enseignement. Comme troisième étape, elle a expérimenté les différentes stratégies sélectionnées puis elle a analysé les résultats obtenus (quatrième étape). Elle n'a donc jamais débuté par l'action, mais par un diagnostic des élèves afin de pouvoir s'adapter rapidement. Puis, elle réinvestit (étape 5) ses observations par rapport aux apprentissages des élèves pour chacune de ses planifications. Bref, elle réalise les cinq étapes du modèle de Korthagen et Vasalos (2005) : comme le soutiennent ces auteurs, l'ordre n'a pas d'importance. 
La pratique réflexive de l'étudiante B s'articule différemment selon le travail demandé, mais surtout selon l'accompagnement offert par ses formateurs :

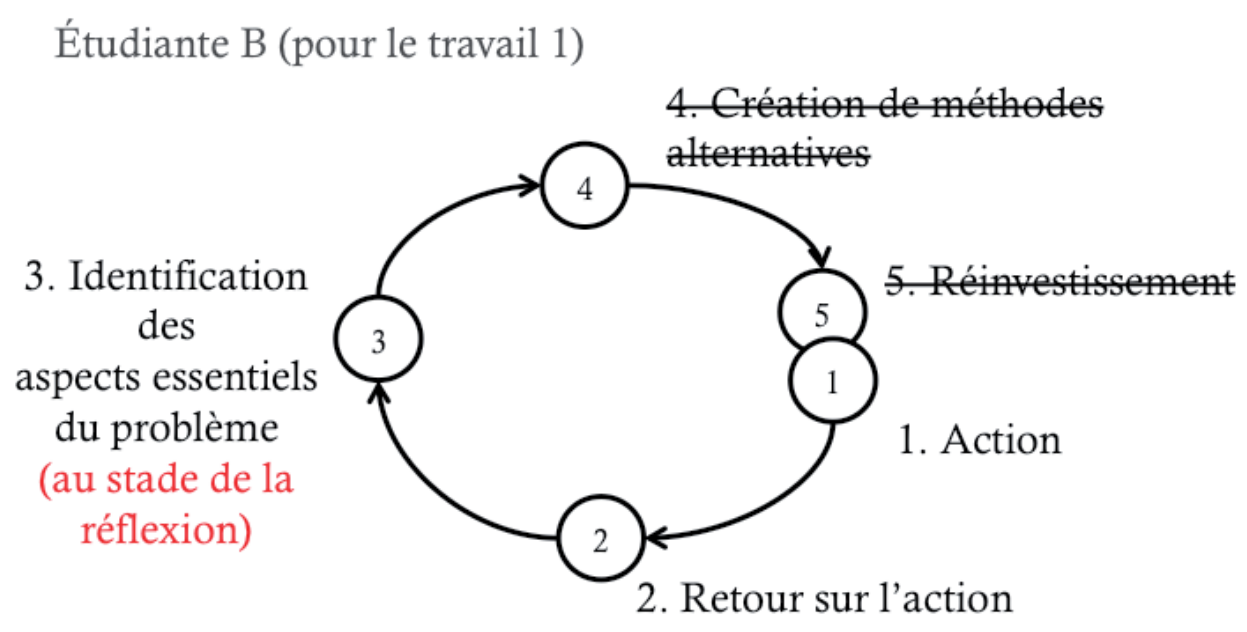

\section{Figure 6}

L'articulation des étapes du modèle ALACT pour l'étudiante B à l'intérieur du travail en didactique.

À l'intérieur du travail d'intégration en didactique du français, l'étudiante B n'a pas fait de diagnostic préalable à propos des compétences des élèves et elle est passée directement à l'action en faisant vivre une activité d'apprentissage. Lors du retour sur l'action, réalisé sans l'aide d'un formateur, elle est difficilement parvenue à identifier les problèmes rencontrés par ses élèves. Elle réfléchit certes aux problèmes rencontrés, mais ne propose aucune méthode ou solution alternative. À l'intérieur du travail sur le forum de discussion animé par sa superviseure de stage, l'étudiante B réalise les cinq étapes du modèle ALACT allant même jusqu'à proposer différentes méthodes alternatives qu'elle réintègre dans de nouvelles situations professionnelles. Elle nomme d'abord un problème :

Je trouve très intéressant que vous [ses pairs] abordiez ce sujet, puisque je me suis beaucoup questionnée quant à la motivation des élèves par rapport aux activités quion leur offre. Dans un groupe en particulier (qui n'a habituellement pas ce problème), il m'a parfois semblé quen dépit des mises en contexte et des situations d'apprentissage qui semblent attrayantes et motivantes, les élèves réagissent mal [...].

Pour trouver des solutions, elle regarde du côté de la littérature :

Ce quien disent les experts - [...] on fait référence à deux professeurs à l'Université de Rochester (Edward L. Deci et Richard M. Ryan) qui soutiennent que la «satisfaction de trois besoins psychologiques considérés comme essentiels joue un rôle déterminant sur la motivation. Il s'agit des besoins d'autonomie, de compétence et de relation avec autrui ». [...] Autrement dit, un élève qui se sent autonome, compétent et soutenu par son entourage aura davantage tendance à persévérer dans ses actions $[\ldots]$. 
Elle propose alors des solutions pour les réinvestir dans sa pratique : "Je donnerai plus de choix aux élèves par rapport aux thèmes, mais également je m'assurerai d'offrir des activités présentant des défis intellectuels. Je vais aussi essayer la classe inversée [...] ». Elle décrit alors la classe inversée et les activités choisies. Cette étudiante profite également du soutien de son enseignant associé qui lui a posé plusieurs questions sur le forum : "Pour quelles raisons choisis-tu cette approche pédagogique? Que veux-tu que les élèrves développent? Quel rôle ont-ils dans cette situation? ». La capacité de l'étudiante stagiaire à réaliser toutes les étapes du modèle ALACT dans ce travail de réflexion collaboratif soulève la question suivante : devrions-nous privilégier les travaux dans lesquels les formateurs font des retours fréquents sur l'action à travers un dialogue (et non uniquement dans une correction à la fin du travail) afin de pousser la réflexion des étudiants? L'étude de cas de l'étudiante B semble appuyer cette piste de formation.

Pour l'étudiante $\mathrm{C}$, les étapes du modèle ALACT se restreignent à l'action et au retour sur l'action pour le travail en didactique et pour le journal professionnel de stage. Dans le travail de didactique, elle voit "l'ensemble du groupe » et ne semble pas en mesure de faire un diagnostic des difficultés des élèves. Elle choisit comme stratégie d'enseignement la prise de notes trouées sans la justifier à l'aide de ce qu'elle a appris dans ses cours de didactique et de gestion de classe. Puisque tout se déroule bien dans la classe lors du pilotage de cette stratégie, elle ne la questionne pas et aucun de ses formateurs (enseignant associé et superviseur universitaire) ne le fait. Ses choix pédagogiques s'appuient essentiellement sur ses croyances : "Je crois que... Je considère que... Moi, je... ». Encore ici, les formateurs n'interviennent pas, ce qui limite son développement professionnel. En effet, aucun recours à des appuis théoriques ou aucune relance ne vient exiger de tels appuis.

À l'intérieur de son journal professionnel, l'étudiante $\mathrm{C}$ explicite une situation de gestion de classe problématique. Elle a imposé une règle de classe aux élèves : elle a refusé l'utilisation des téléphones cellulaires en classe contrairement à son enseignante associée qui les permettait. Elle remarque que l'établissement et l'exécution de cette règle lui apportent beaucoup de problèmes de gestion de classe (climat de classe, comportements inappropriés des élèves). En aucun temps elle ne remet en question la règle et son processus d'implantation : elle cherche uniquement à être plus "sévère à l'avenir ". Elle ne fait aucun lien théorique avec le cours de gestion de classe et les processus de mises en œuvre de règles de classe. Elle ne semble pas prendre conscience de l'impact de ses choix professionnels (Korthagen, 2010), peut-être parce que son superviseur universitaire n'a pas pu l'accompagner dans son analyse et que la correction se réalisait après la remise du journal. Puisqu'elle avait respecté les étapes réflexives du travail - explicitation de la situation, analyse et pistes de réinvestissement -, elle a obtenu un excellent résultat. Par contre, elle n'a utilisé aucun appui scientifique pour justifier le choix et le processus d'implantation de la règle de classe dans son analyse. Dans le forum de discussion, elle réalise les cinq étapes du modèle ALACT en prenant conscience de ses actions professionnelles possiblement grâce à ses collègues qui la questionnent et la relancent. Cela réaffirme encore l'importance de la relance par autrui pour amener les étudiants-stagiaires plus loin dans leur pratique réflexive, plutôt que d'obtenir une rétroaction seulement à la fin du processus (Korthagen, 2010).

L'étudiante D exploite dans l'ordre les cinq étapes du modèle ALACT dans son journal professionnel et dans le forum : action, retour sur l'action, identification des problèmes, création de méthodes alternatives et réinvestissement. Toutefois, pour le travail en didactique, les quatrième et cinquième étapes sont absentes : 
J'ai décidé de faire une brève présentation théorique de façon magistrale en lien avec le résumé [...]. La théorie que j’ai présentée visait à leur expliquer [aux élèves] à quoi sert un résumé [...], ce qu'est un bon résumé et comment en faire un. [...] Les élèves ont eu de la difficulté à commencer leur résumé. [....] Je ne crois pas que leur incompréhension était liée à un problème de gestion de classe [...]. Je ne crois pas non plus qu'il faut condamner l'enseignement magistral, mais il y a certainement des éléments à améliorer.

Le fait qu'elle ne propose pas de méthodes alternatives (quatrième étape du modèle ALACT) est peut-être causé par l'absence d'accompagnement du didacticien qui corrige le travail après sa remise. Il ne lui pose donc pas de questions qui pourraient lui permettre de progresser dans sa réflexion, telles que, par exemple : pour quelles raisons les élèves éprouvent-ils des difficultés à amorcer leur travail? Quelles stratégies d'enseignement et d'apprentissage auraient pu aider les élèves? Encore ici, le jugement de l'étudiante $\mathrm{D}$ est appuyé sur des croyances. Peu de ces croyances sont en lien avec des référents théoriques.

\section{Discussion}

À l'intérieur des trois travaux analysés, les savoirs scientifiques sont absents tandis que les savoirs académiques et de vulgarisation sont les plus fréquents. La recherche scientifique occupe donc une place marginale dans la pratique réflexive des étudiants-stagiaires, probablement parce que les savoirs étudiés dans les cours universitaires sont davantage vulgarisés pour répondre aux besoins des étudiants sans réel lien avec la recherche scientifique et les publications qui en découlent. Les savoirs prescrits qui sont principalement liés aux compétences professionnelles des enseignants émergent beaucoup en didactique. La formulation des consignes du travail explique peut-être ce résultat puisque les étudiants devaient faire des liens avec les compétences professionnelles. Il demeure que les types de savoirs auxquels font appel les étudiants sont assez peu variés dans les trois travaux, et ce, même si chaque grille d'évaluation contenait des critères pour faire des liens avec les contenus des cours universitaires.

L'étudiante B à l'intérieur de deux travaux sur trois prend conscience de son activité enseignante. Sans l'aide de ses formateurs, elle n'a pas le « réflexe » de se questionner, écrira-t-elle dans une contribution. Ce constat nous a amenés à jeter un regard critique sur les consignes des trois travaux. Nous ne pouvons pas nous assurer d'un processus réflexif chez les étudiants-stagiaires si un cadre théorique de la pratique réflexive n'est pas proposé et piloté par les formateurs pour la réalisation des travaux liés au stage. Le plaquage d'un cadre théorique pour soutenir la rédaction (journal professionnel) ou une consigne générale comme celle à l'intérieur du travail en didactique : " de revenir de manière réflexive sur vos interventions dans la perspective de la didactique de votre discipline » n'est pas suffisante pour guider les étudiants-stagiaires dans un véritable processus réflexif. Cette absence de cadre théorique pour soutenir la pratique réflexive soulève un autre défi : amener les étudiants-stagiaires à s'appuyer sur des savoirs de référence et non principalement sur des savoirs intuitifs et expérientiels pour étayer leur jugement professionnel exigera de s'asseoir avec l'ensemble des formateurs universitaires, superviseurs et professeurs, afin de s'entendre sur ces exigences. 
Le type de travail demandé en stage soulève une interrogation : limitons-nous la pratique réflexive des étudiants-stagiaires en les obligeant à composer un journal professionnel qui propose un cadre réflexif par étapes, annoté une fois terminé, et sur lequel aucun retour n'est proposé? Ce genre d'écrit monologique ne favorise pas l'apport des formateurs et des pairs; nous remarquons un manque d'approfondissement dans l'analyse des étudiantes parce qu'elles ne bénéficient pas d'étayage. Les étudiantes-stagiaires restent donc dans une phase naïve de compréhension et d'analyse qui est non alimentée par des savoirs théoriques. Si elles étaient mieux accompagnées dans ce travail par leurs formateurs et leurs pairs, qui commenteraient pour les aider à bonifier leurs réflexions et leurs assises théoriques, elles pourraient jeter un nouvel éclairage sur leur action. Pour le moment, plusieurs comportements et aspects de leur pratique restent inconscients pour elles. En analysant les contributions sur le forum de coélaboration de connaissances, ce sont les apports externes des pairs et des formateurs qui ont permis aux étudiantesstagiaires d'aller plus loin dans leurs réflexions en mettant à distance leur pratique à l'aide de savoirs construits par la recherche. Certes, les savoirs scientifiques demeurent marginaux dans le forum de discussion, comme dans tous les autres travaux demandés, mais le potentiel de développement de la pensée appuyée par les relances des différents formateurs et des pairs ouvre une piste pour le renforcement de l'articulation théorie-pratique en formation à l'enseignement (Butke, 2006; Mann et al., 2009).

\section{Conclusion}

En ce sens, cette étude soulève des questions centrales dans le développement de la pratique réflexive des étudiants-stagiaires : pour quelles raisons les savoirs scientifiques sont-ils si peu présents dans leur discours? Les cours universitaires favorisent-ils la mobilisation de sources de savoirs variés? Le soutien que leur apportent leurs formateurs en stage, la forme des travaux et les questions qui s'y retrouvent favorisent-ils le développement de cette pensée réflexive? Il ne s'agit pas d'une recherche qui permette la généralisation des résultats puisque seulement trois travaux ont été analysés et qu'un nombre restreint d'étudiantes-stagiaires a été sélectionné. Cependant, à la lumière des données analysées, nous sommes enclins à penser que les travaux qui permettent un dialogue, une forme de relance autour des réflexions des stagiaires, sont les plus susceptibles de permettre à ces derniers de dépasser un niveau assez général de pratique réflexive basé sur une analyse intuitive. Mais se pose alors la question de la formation des formateurs : les pédagogues formateurs sont-ils en mesure d'accompagner les étudiantsstagiaires à travers les différents stades d'analyse réflexive? Pour peu que les institutions de formation leur proposent des cadres théoriques cohérents et opérationnels pour soutenir le développement de la pratique réflexive. Nous pensons qu'une analyse comme celle que nous venons de proposer pose les bases d'une réflexion à l'égard des cadres théoriques à mobiliser auprès des étudiants et des formateurs.

\section{Notes}

1 Problème : questionnement personnel sur une situation exemplaire ou difficile.

2 Formalisés : verbalisés de manière structurée. 


\section{Références}

Avalos, B. (2011). Teacher professional development in Teaching and Teacher Education over ten years. Teaching and Teacher Education, 27(1), 10-20. http://dx.doi.org/10.1016/j.tate.2010.08.007

Bullough, R. V. et Draper, R. J. (2004). Making sense of a failed triad : Mentors, university supervisors, and positioning theory. Journal of Teacher Education, 55(5), 407-420. http://dx.doi.org/10.1177/0022487104269804

Butke, M. A. (2006). Reflection on practice: A study of five choral educators' reflective journeys. Update: Applications of Research in Music Education, 25(1), 57-69. http://dx.doi.org/10.1177/87551233060250010107

Buysse, A. A. J. (2011). Une modélisation des régulations et de la médiation dans la construction des savoirs professionnels des enseignants. Dans P. Maubant, et S. Martineau (dir.), Fondements des pratiques professionnelles des enseignants (p. 243-284). Ottawa, ON : Presses universitaires d'Ottawa.

Calderhead, J. (1989). Reflective teaching and teacher education. Teaching and Teacher Education, 5(1), 43-51. http://dx.doi.org/10.1016/0742-051x(89)90018-8

Clarke, A. (2006). The nature and the substance of cooperating teacher reflection. Teaching and Teacher Education, 22(7), 910-921. http://dx.doi.org/10.1016/j.tate.2006.04.039

Clarke, A. et Collins, S. (2007). Complexity science and student teacher supervision. Teaching and Teacher Education, 23(2), 160-172. http://dx.doi.org/10.1016/j.tate.2006.10.006

Comité d'agrément des programmes de formation à l'enseignement (CAPFE). (2015). Bilan des visites de suivi-Regard sur l'état actuel de la formation à l'enseignement au Québec. Repéré à

http://www.education.gouv.qc.ca/fileadmin/site web/documents/reseau/formation titularisation/Bilan visites suivi.pdf

Desbiens, J.-F., Spallanzani, C. et Borges, C. (2013). Quand le stage en enseignement déraille : regards pluriels sur une réalité trop souvent occultée. Québec, QC : Presses de l'Université du Québec.

Dewey, J. (1938). Logic - The theory of inquiry. New York, NY : Holt.

Donnay, J. et Charlier, E. (2006). Apprendre par l'analyse de pratiques. Initiation au compagnonnage réflexif. Namur : Presses universitaires de Namur.

Harrison, J., Dymoke, S. et Pell, T. (2006). Mentoring beginning teachers in secondary schools: An analysis of practice. Teaching and Teacher Education, 22(8), 1055-1067. http://dx.doi.org/10.1016/j.tate.2006.04.021

Jay, J. K. et Johnson, K. L. (2002). Capturing complexity: a typology of reflective practice for teacher education. Teaching and Teacher Education, 18(1), 73-85. http://dx.doi.org/10.1016/s0742-051x(01)00051-8

Korthagen, F. A. J. (1985). Reflective teaching and preservice teacher education in the Netherlands. Journal of Teacher Education, 36(5), 11-15. http://dx.doi.org/10.1177/002248718503600502

Korthagen, F. A. J. (2010). How teacher education can make a difference. Journal of Education for Teaching, 36(4), $407-423$. http://dx.doi.org/10.1080/02607476.2010.513854

Korthagen, F. A. J. (2016). Pedagogy of teacher education. Dans J. Loughran et M. L. Hamilton (dir.), International Handbook of Teacher Education (vol. 1, p. 311-346). Singapour : Springer.

Korthagen, F. A. J. et Vasalos, A. (2005). Levels in reflection: Core reflection as a means to enhance professional development. Teachers and Teaching: Theory and Practice, 11(1), 47-71. http://dx.doi.org/10.1080/1354060042000337093

Krippendorff, K. (2004). Content analysis: An introduction to its methodology (2 éd.). Thousand Oaks, CA : SAGE.

Mann, K., Gordon, J. et MacLeod, A. (2009). Reflection and reflective practice in health professions education: a systematic review. Advances in Health Sciences Education, 14(4), 595-621. http://dx.doi.org/10.1007/s10459-007-9090-2

Miles, M. B. et Huberman, A. M. (2003). Analyse des données qualitatives (2e e冈d.). Paris : De Boeck. 
Ministère de l'Éducation du Québec (MEQ). (2001). La formation à l'enseignement - Les orientations - Les compétences professionnelles. Repéré à http://www.education.gouv.qc.ca/fileadmin/site web/documents/reseau/formation titularisation/formation enseignement orientations EN.pdf

Portelance, L., Gervais, C., Lessard, M. et Beaulieu, P. (2008). La formation des enseignants associés et des superviseurs universitaires. Rapport de recherche. Cadre de référence. Repéré à https://www.usherbrooke.ca/education/fileadmin/sites/ education/documents/Intranet/Documents officiels/Rapport Cadre reference 2009.pdf

Rogers, B. (dir.). (2002). Teacher leadership and behaviour management. Thousand Oaks, CA : SAGE. http://dx.doi.org/10.4135/9781446214954

Scardamalia, M. (2004). CSILE/Knowledge forum ${ }^{\circledR}$. Dans A. Kovalchick (dir.), Education and technology: An encyclopedia (p. 183-192). Repéré à http://www.ikit.org/fulltext/CSILE KF.pdf

Schön, D. A. (1983). The reflective practitioner: how professionals think in action. New York, NY : Basic Books.

Serres, G., Ria, L. et Adé, D. (2004). Modalités de développement de l'activité professionnelle au gré des contextes de classe et de formation : le cas des professeurs stagiaires en éducation physique et sportive. Revue française de pédagogie, (149), 49-64. http://dx.doi.org/10.3406/rfp.2004.3172

Sharples, M. (2005). Learning as conversation transforming education in the mobile age. Repéré à https://pdfs.semanticscholar. org/b127/35e47551f62ef22c957e55781148f68fcbd0.pdf

Tardif, M. et Jobin, V. (2014). Les compétences en formation à l'enseignement : bilan critique de vingt ans de réformes québécoises. Dans M. Tardif et J.-F. Desbiens (dir.), La vogue des compétences dans la formation des enseignants : bilan critique et perspectives d'avenir. (p. 61-86). Québec, QC : Presses de l'Université Laval.

\section{Pour citer cet article}

Gouin, J.-A., Hamel, C. et Falardeau, É. (2018). L'analyse des savoirs mobilisés par des étudiantes-stagiaires en enseignement dans leur pratique réflexive. Formation et profession, 26(2), 18-34.

http://dx.doi.org/ 10.18162/fp.2018.454 